\title{
Aprender Mecánica de Vuelo Orbital mediante problemas y simulaciones con STK10
}

Santiago E. Moll, José A. Moraño y Luis M. Sánchez Ruiz

Dpto. de Matemática Aplicada, Universitat Politècnica de València, Spain, \{sanmollp,jomofer,lmsr\}@mat.upv.es

\begin{abstract}
The School of Design Engineering at the Polytechnic University of Valencia has begun to teach this course 2014-2015 the new Master in Aeronautical Engineering. In the first year the compulsory subject 'Orbital and Atmospheric Flight Mechanics. Guidance Laws' appears with 4.5 ECTS (Theory 2.25, Practice 1.25 and Problems with computer 1.00).

Developing the curriculum of this course has been the first step in the work of the teachers involved, who have divided the competences of the subject in two blocks. On the one hand the competences of 'Orbital and Atmospheric Flight Mechanics' and on the other those of 'Guidance Laws'.

It has also sought to promote a type of learning based on problems connected with reality as well as allowing students to visualize the elements that they are studying. Each weekly session consists of an introduction of concepts in theoretical session, a problem-solving task to strengthen and expand knowledge and finally a visualization practice by performing $2 D$ and $3 D$ simulations by means of STK software.

In this article we describe the process followed in developing the curriculum of the first block (MVOyA) showing also some examples of the material used, the results obtained and the students' opinion.
\end{abstract}

Keywords: Simulation-based learning, Orbital Mechanics, STK.

\section{Resumen}

La Escuela Técnica Superior de Ingeniería del Diseño de la Universitat Politècnica de València ha comenzado a impartir este curso 2014-2015 el Máster en Ingeniería Aeronáutica. En primer curso aparece la asignatura 
Aprender Mecánica de Vuelo Orbital mediante problemas y simulaciones con STK10

obligatoria 'Mecánica de vuelo orbital y atmosférico. Leyes de guiado' con 4,5 ECTS (Teoría 2,25, Problemas 1.25 y Prácticas con ordenador 1.00).

Elaborar el currículum de esta asignatura ha sido el primer paso en el trabajo de los profesores implicados, que han dividido las competencias de la asignatura en dos bloques. Por una parte las competencias de 'Mecánica de Vuelo Orbital y Atmosférico' y por otra las de 'Leyes de Guiado'.

También se ha pretendido potenciar un tipo de aprendizaje basado en problemas conectados con la realidad además de permitir que los alumnos puedan visualizar los elementos que van estudiando. Cada sesión semanal consta de una introducción de conceptos en la sesión teórica, una tarea de resolución de problemas para reforzar y ampliar conocimientos y finalmente de una práctica de visualización mediante la realización de simulaciones $2 D$ y $3 D$ con STK10.

Aquí describimos el proceso seguido en la elaboración del currículum del primer bloque (MVOyA) y presentamos ejemplos del material utilizado, resultados obtenidos y la opinión del alumnado.

Palabras clave: Simulaciones con STK, Dinámica orbital, Mecánica de vuelo orbital

\section{Introducción}

El Vicerrectorado de Estudios, Calidad y Acreditación (VECA) de la Universitat Politècnica de València (UPV) a través del Instituto de Ciencias de la Educación (ICE) realiza cada año una convocatoria de ayudas para la realización de Proyectos de Innovación y Mejora Educativa (PIME). Durante el curso 2014-2015 los profesores de la asignatura de “Mecánica de Vuelo Orbital y Atmosférico. Leyes de guiado” del Máster en Ingeniería Aeronáutica que se imparte en la Escuela Técnica Superior de Ingeniería del Diseño (ETSID), están diseñando y ejecutando el currículum de esta nueva asignatura dentro del proyecto PIME B-25.

La asignatura se imparte en el segundo semestre del Máster con un total de 4,5 ECTS de los que 2,25 corresponden a Teoría (TA), 1,25 a Problemas (PA) y 1,00 a Prácticas con ordenador (PL). Para el proceso de elaboración del currículum el conjunto de competencias de la asignatura ha sido dividido en dos bloques, por una parte las competencias de 'Mecánica de Vuelo Orbital y Atmosférico' (MVOyA) y por otra, las correspondientes a 'Leyes de Guiado'. Cada bloque tiene 7 sesiones de TA de 90’ que se imparten cada 
semana y otras 7 sesiones de 90' también semanales, con una primera parte de corrección, revisión y comentario de problemas realizados en tareas semanales, PA, y una segunda parte, PL, de realización de una actividad práctica. En el bloque MVOyA las prácticas con ordenador se realizan con el apoyo del software científico System Tool Kit (STK). La distribución del tiempo para cada parte de estas sesiones PA/PL no esta fijada pero suele ser de 30' y 60' para cada una de las partes respectivamente.

Se hecho un diseño del currículum intentando combinar tanto clases magistrales, como tareas que fomentan el aprendizaje basado en problemas y prácticas mediante simulaciones 2D y 3D apoyadas en datos reales (Tiana, 2015). En las clases magistrales de las sesiones TA se presentan los conocimientos básicos necesarios para poder realizar los problemas propuestos en las tareas donde aparecen junto a los conceptos básicos anteriores otros que el alumno debe conocer para conseguir el nivel de competencia deseado en la sesión. Además para las sesiones de PL en las que se trabaja con ordenador se han diseñado unas prácticas donde el alumno descubre relaciones de elementos y conceptos de la asignatura con la realidad mediante la manipulación de parámetros en simulaciones 2D y 3D realizadas con el software científico STK.

\section{Objetivos}

Los objetivos de este trabajo son por tanto:

- Desarrollar el nuevo currículum de la asignatura y en particular del bloque MVOyA

- Diseñar las sesiones TA y PA+PL de forma que las competencias de cada semana estén conectadas entre sí:

- Ajustando los contenidos teóricos a los 90’ de cada sesión

o Preparando uno o varios problemas que fuercen un repaso de la teoría y que fomenten una ampliación de esos conocimientos

o Proyectando actividades para realizar simulaciones que permitan al alumno entender, visualizar y analizar los conceptos de esa semana.

Estos objetivos persiguen por tanto desarrollar una metodología activa mediante la realización de simulaciones con STK.

\section{Combinando conceptos, problemas y simulaciones}

Los contenidos del bloque de MVOyA se han organizado en 7 semanas donde para cada una se ha diseñado una combinación de tres metodologías. Se utiliza un aprendizaje de contenidos mediante clases presenciales para la explicación de conceptos y procedimientos

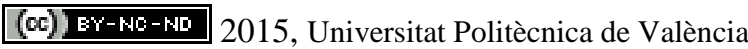


Aprender Mecánica de Vuelo Orbital mediante problemas y simulaciones con STK10

necesarios para poder desarrollar las competencias programadas esa semana. Este aprendizaje se combina con una instrucción basada en la resolución de problemas que permitan, además de aplicar esos conocimientos, entender y aprender posibles extensiones de los mismos. Se completa esta combinación con la realización de una práctica con ordenador en la que se propone al alumno una o varias simulaciones para llegar a visualizar las diferentes situaciones con las que puede llegar a encontrarse al trabajar con estas competencias. Con las simulaciones realizadas mediante el uso del software científico STK se pretende impulsar la actitud analítica del estudiante (Heid, 2008, Tall, 2000), al tiempo que se fuerza el repaso de ciertos conceptos y habilidades y su aplicación de manera eficiente a un mundo cuasi-real, ya que este objetivo es más fácil de lograr si los estudiantes trabajan persiguiendo el efecto 'hacer y entender' por medio de simulaciones que sólo con la resolución de problemas (Tiwari, 2014). Para esto es necesario que las simulaciones estén bien sincronizadas con la aparición de otros contenidos dentro de la asignatura para garantizar así que el proceso de aprendizaje no se haga de forma interrumpida.

El software STK y sus simulaciones se pueden utilizar, y así se ha hecho, para diferentes propósitos en MVOyA. En las próximas subsecciones se muestran algunos ejemplos.

\subsection{Las simulaciones son útiles para revisar y comprender los conceptos teóricos}

El software STK ha demostrado ser muy útil para ayudar a la comprensión de los conceptos vistos en teoría, como por ejemplo: cambios de coordenadas, elementos orbitales, traza de los satélites, etc. El proceso seguido es el siguiente, dentro de una sesión TA se explican los diferentes tipos de coordenadas que existen, cómo caracterizan el movimiento de un satélite sus elementos orbitales, la relevancia que tiene el periodo orbital en ese movimiento, etc. Al final de dicha sesión se propone al alumno la resolución de algún problema de cambio de coordenadas y de cálculo de efemérides con los elementos orbitales. En estos problemas se aplica lo visto en las sesiones de TA pero también se añaden conceptos nuevos que permiten abordar un problema más avanzado como, por ejemplo en este caso, saber hallar la proyección sobre la superficie terrestre de la posición de un satélite en cada instante (concepto conocido como traza). Finalmente planteamos al alumno una práctica con la creación de un escenario que incorpore varios satélites definiendo ellos mismos sus propiedades mediante la ventana 'Define Properties'. Se les proporcionan los pasos necesarios para activar el movimiento de la simulación que se puede visualizar en 3D y de esta manera se les permite diferenciar entre algunos tipos de órbitas (polar, geoestacionaria, con y sin inclinación, etc) según sus elementos orbitales (ver Figura 1a). También podemos visualizar esta simulación en la ventana 2D para entender el concepto de traza y cómo los elementos orbitales afectan a su representación, como por ejemplo, el periodo, la 
inclinación, la ascensión recta del nodo ascendente $(\Omega)$, el perigeo y apogeo , etc. (ver Figura 1b).
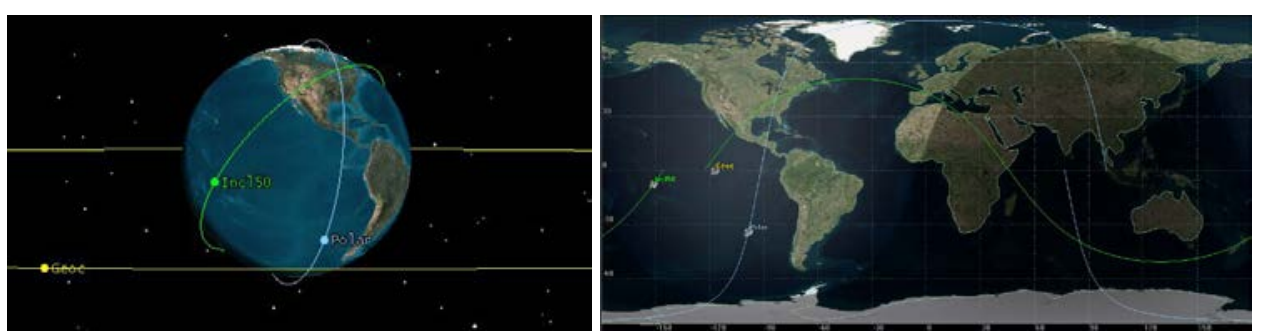

Fig. 1. (a) Representación 3D de tres tipos de órbitas. (b) Visualización 2D de las trazas de los tres satélites.

\subsection{Las simulaciones permiten hacer animaciones de largos periodos de tiempo}

Otra valor destacado de STK es la posibilidad de realizar simulaciones de largos periodos de tiempo para detectar errores que no se perciben en las simulaciones en tiempo real. Existen características que se observan en la teoría de MVOyA que a priori parecen menos importantes, pero que cuando se realizan simulaciones para mucho tiempo pasan a ser notables, por ejemplo la diferencia entre día sidéreo y día civil. En la sesión de TA se definen los diferentes tiempos que se utilizan en astronomía pero resulta complicado ver qué efecto puede causar esta diferencia en el movimiento de un satélite. Tras la introducción teórica se propone una actividad sobre un satélite geoestacionario cuyo periodo orbital sea el día sidéreo y el civil. Para la sesión de PA+PL se le propone al alumno la creación de un escenario de STK con la inserción de 2 objetos geoestacionarios (satélites) que en esta ocasión se pueden introducir por medio del asistente 'Orbit Wizard'. A continuación se recomienda al alumno modificar el periodo de uno de los objetos a 24 horas exactamente y se le sugiere que desplace la barra de tiempo de simulación para que pueda observar que el satélite pierde la cualidad de geoestacionario (ver Figuras 2a y 2b).
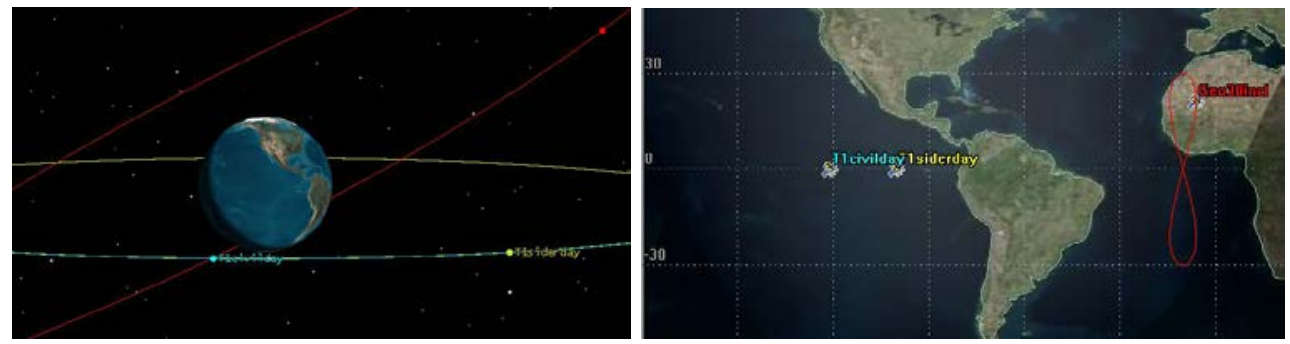

Fig. 2. (a) Visualización 3D del retraso acumulado en varios días por el satélite cuyo periodo es 1 día civil (azul) respecto de uno geoestacionario (amarillo). (b) Visulización 2D de la misma simulación.

(c)) EY-NC-ND 2015, Universitat Politècnica de València

Congreso In-Red (2015) 
Aprender Mecánica de Vuelo Orbital mediante problemas y simulaciones con STK10

\subsection{STK permite realizar simulaciones con objetos reales desde su base de datos}

El programa STK ofrece la posibilidad de hacer simulaciones con objetos reales que pueden incorporarse directamente desde su base de datos. Con ellos podemos mostrar al alumno efectos reales que se producen por pequeñas perturbaciones que con el tiempo se van acumulando, en particular, nosotros hemos utilizado esta ventaja para mostrar al alumno la perturbación que el achatamiento terrestre provoca sobre ciertas órbitas. En la sesión TA correspondiente se explican las perturbaciones y en particular las del efecto J2 o achatamiento terrestre y luego se proponen un par de ejercicios para su aplicación. Sin embargo tener una intuición de cómo esto altera el movimiento de un satélite resulta difícil sin la simulación. Para trasladar al alumno esa intuición se prepara una práctica para PA+PL partiendo de uno de los muchos objetos de la base de datos de STK, la Estación Espacial Internacional (ISS).

Con los elementos obtenidos de la ISS, debe crear dos satélites, pero para la simulación debe incorporar a uno de ellos la perturbación debido al achatamiento terrestre mientras que al otro objeto le mantiene una integración de tipo 'Two Body' fija. Cuando amplia el intervalo de visualización el alumno podrá comprobar los movimientos de ambos satélites y el desplazamiento que el efecto J2 ha provocado en $\Omega$ (ver Figuras 3a y 3b). En ese momento se pide que recuerden la expresión del movimiento angular que genera esta deformación, estudiado en la teoría, con el fin de corroborar el efecto del retraso de la ascensión recta del nodo ascendente (RAAN) de la ISS por la perturbación.
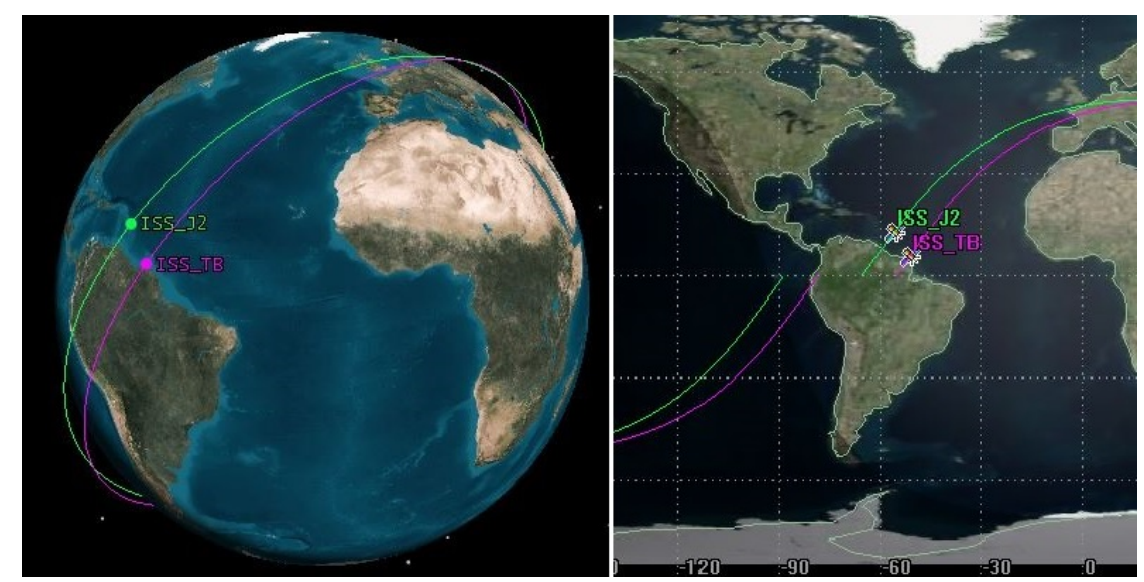

Fig. 3. Imágenes $3 D$ (a) y 2D (b) de la simulación de las órbitas de la ISS considerando (ISS_J2) o no (ISS_TB) el efecto del achatamiento terrestre. 


\subsection{La simulación puede ser utilizada para corroborar cálculos}

En ocasiones se puede utilizar el software simulador para confirmar que los cálculos realizados para una determinada situación pueden ser reales. En MVOyA se ha utilizado esta cualidad para confirmar las características de algunas órbitas especiales. Por ejemplo, en la sesión de TA correspondiente se presentan cierto tipos de órbitas como tipo Molniya, Tundra, polar, heliosíncrona, etc. Algunos elementos de estas órbitas deben tener ciertos valores para mantener sus propiedades. Tras los ejercicios de cálculo que se proponen al final de la sesión TA se propone una sesión práctica donde el alumno crea un escenario con un satélite cuyos elementos son los de uno de tipo Molniya salvo la $i\left(i \neq 63.4^{\circ}\right)$. Ejecutando la simulación un breve periodo, el satélite funciona dando la cobertura como se espera. Pero al ampliar el intervalo de tiempo a 2 años se aprecia en ambas ventanas 2D y 3D que el perigeo de la órbita original, se ha convertido en el apogeo y viceversa (ver Figura 4).

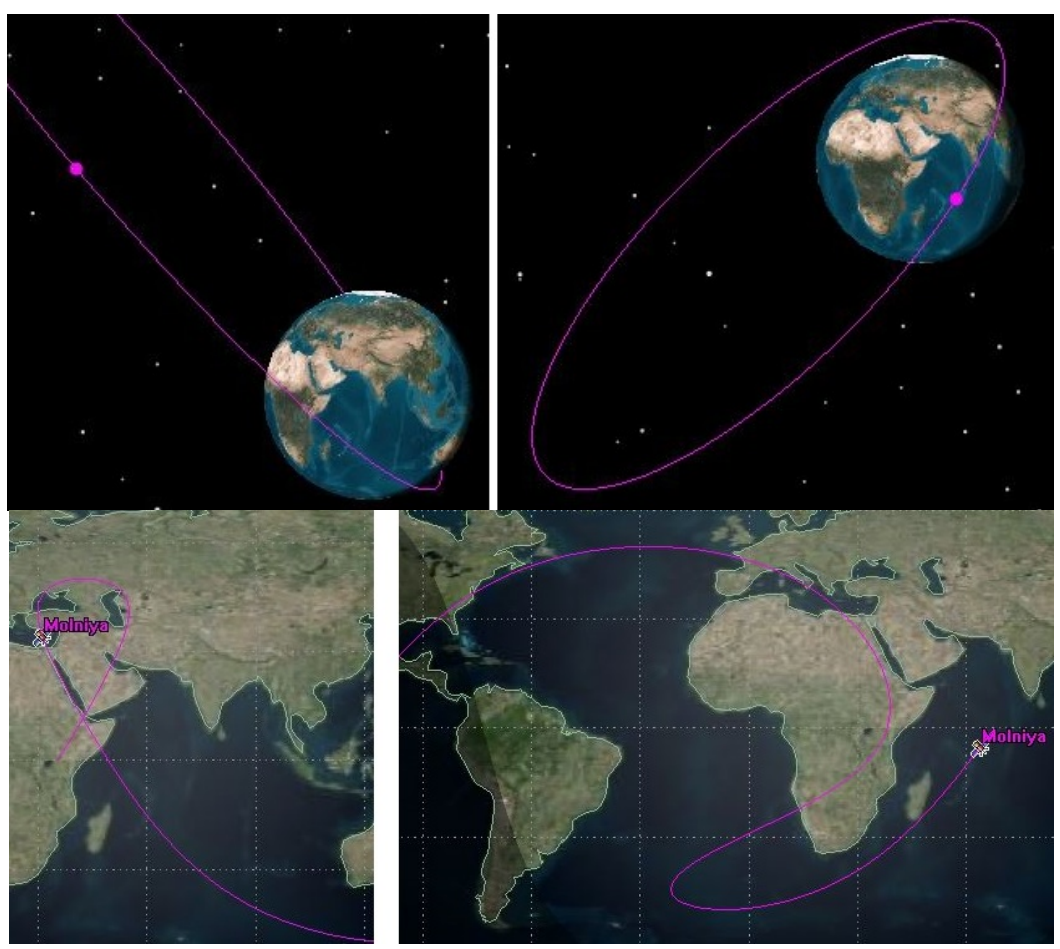

Fig. 4. Si $i \neq 63.4^{\circ}$ el efecto J2 cambia el perigeo de la órbita con el paso del tiempo.

En ese momento se pide al alumno que recuerde que la razón de esta alteración es el efecto $\mathrm{J} 2$ sobre el argumento del perigeo $(\omega)$ y que encuentren el valor de $i$ que elimina esta perturbación. Esto motiva a los estudiantes a revisar los conocimientos y las fórmulas para llegar a que $\mathrm{i}=63.4^{\circ}$. Ahora se pide la modificación de este elemento de la órbita en la simulación que permitirá ver que la posición del apogeo no cambia con el tiempo. 
Aprender Mecánica de Vuelo Orbital mediante problemas y simulaciones con STK10

\subsection{STK permite generar informes y gráficas sobre la funcionabilidad de un proyecto}

A veces la utilidad de una simulación está en los informes o gráficos que genera y que nos permiten comprobar si un proyecto cumple con las expectativas para las que se crea. Este rasgo se ha utilizado en MVOyA para ver si un proyecto de constelación de satélites dan la cobertura y visibilidad requeridas para un lugar. Como siempre los conceptos y métodos para calcular cobertura y visibilidad se presentan en una sesión TA tras la cual se propone una actividad con la creación de una constelación de satélites. Con el fin de consolidar este conocimiento se presenta una práctica de simulación en la que el alumno coloca 3 satélites en una órbita Molniya y una base como por ejemplo Baikonur. Después hay que introducir sensores en los satélites y para verificar si sus satélites proporcionan una cobertura total. Se muestra una herramienta útil de STK (Graphics - Access) que representa la cobertura de los sensores. La gráfica de la simulación permite confirmar o descartar si los ángulos estimados teóricamente para visibilidad y cobertura entre la instalación y el satélite son adecuados (ver Figura 5).

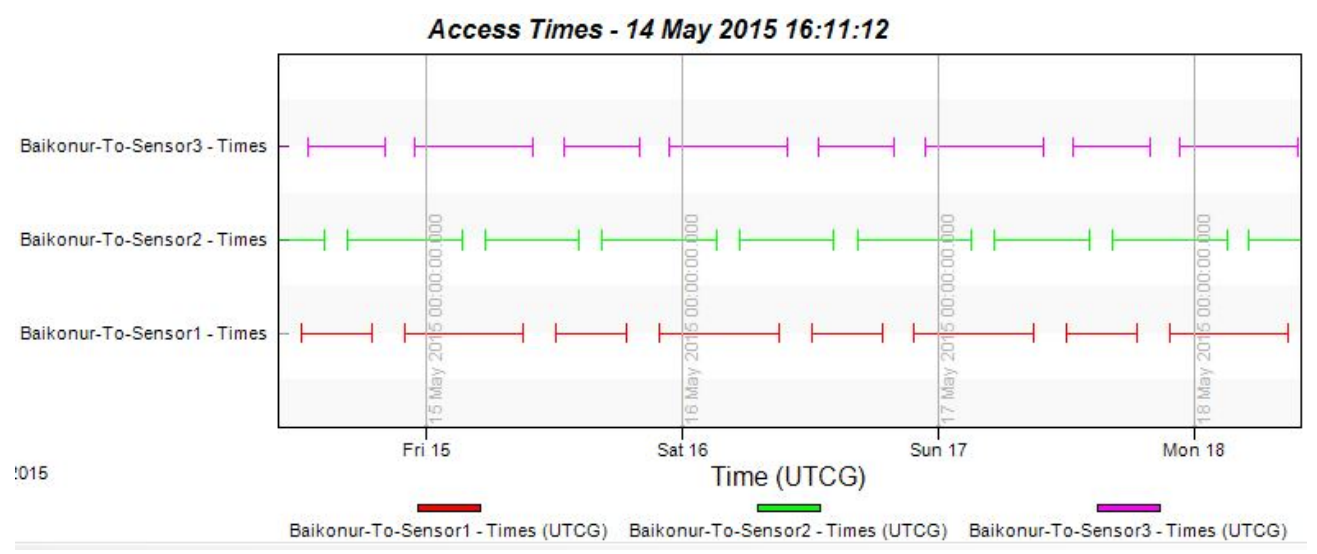

Fig. 5 Ejemplo de un gráfico de la cobetura de tres satélites

\section{Resultados del trabajo: Calificaciones y Opiniones}

\subsection{Calificaciones}

Para la evaluación de los alumnos en el bloque de MVOyA se han considerado tres tipos de calificaciones siguiendo con lo establecido en la guía docente de la asignatura: En primer lugar se han obtenido las notas de los ejercicios propuestos al acabar cada sesión de TA $\left(\mathrm{N}_{-} \mathrm{T}\right)$, junto con estas se han recogido las calificaciones de las prácticas realizadas en las sesiones PA+PL (N_P) y además se ha recogido una nota de una prueba presencial (N_Ex) realizada al acabar el temario. Los resultados obtenidos son presentados en la Tabla 1. 
Tabla 1. Calificaciones del bloque de MVOyA

\begin{tabular}{ccccc}
\hline & $<5$ & [5-7[ & [7-9[ & [9-10] \\
\hline N_T (30\%) & 1 & 1 & 3 & 41 \\
N_P (30\%) & 1 & 0 & 2 & 43 \\
N_Ex (40\%) & 2 & 11 & 25 & 8 \\
Nota & 1 & 1 & 31 & 13 \\
\hline
\end{tabular}

Los resultados obtenidos son significativamente buenos, ya que la tasa de abandono ha sido de 2,2\% (1/46) y la tasa de éxito del 97,8\% (45/46), incluso para un curso de máster. Cabe señalar que hubo un único estudiante que no hizo el examen y que en realidad sólo ha asistido a las sesiones durante las dos primeras semanas.

\subsection{Sondeos}

Después de las $7+7$ sesiones y la prueba de evaluación en el aula, se consultó a los estudiantes acerca de sus opiniones sobre las simulaciones utilizadas en el bloque MVOyA. En las Tablas 2 y 3 podemos ver cuestiones planteadas y la distribución de las respuestas.

Tabla 2. Considero que usar STK10 en las clases de Prácticas de MVO es...

\begin{tabular}{cccc}
\hline Prescindible & Recomendable & Indispensable & NS/NC \\
\hline $0 \%$ & $36 \%$ & $64 \%$ & $0 \%$ \\
\hline
\end{tabular}

Tabla 3. ¿Consideras que utilizar un simulador de órbitas como STK10 ha ayudado a comprender términos y conceptos de las clases teóricas?

\begin{tabular}{ccccr}
\hline Nada & Poco & Bastante & Mucho & NS/NC \\
\hline $0 \%$ & $4 \%$ & $16 \%$ & $80 \%$ & $0 \%$ \\
\hline
\end{tabular}

Se puede observar que para la mayoría de los estudiantes el uso de STK10 es esencial para el aprendizaje de las competencias de este bloque y les ayuda mucho para la comprensión de términos y conceptos teóricos. Las consultas se llevaron a cabo de forma anónima con la herramienta ‘Sondeos' de la plataforma educativa desarrollada por la UPV (PoliformaT).

\section{(c)) EY-NC-ND 2015, Universitat Politècnica de València}


Aprender Mecánica de Vuelo Orbital mediante problemas y simulaciones con STK10

\section{Conclusion}

El software STK se ha integrado en la metodología educativa del bloque de Mecánica de Vuelo Orbital y Atmosférico para realizar simulaciones que, tal y como como se muestra en este trabajo, han facilitado el aprendizaje. Las simulaciones junto con los problemas han permitido alcanzar a nuestros estudiantes las competencias y habilidades exigidas de forma satisfactoria. Al mismo tiempo el programa STK ha permitido relacionar los contenidos teóricos con el entorno real. Los resultados obtenidos han sido excelentes con sólo un abandono ocurrido la primera semana del curso. La opinión de los estudiantes también ha sido recabada, siendo ésta muy positiva.

\section{Agradecimientos}

Los autores agradecen al Departamento de Matemática Aplicada de la UPV por el establecimiento de ayudas a proyectos educativos innovadores, a la ETSID por su apoyo continuo en los proyectos de innovación docente y al Vicerrectorado de Estudios y Convergencia Europea de la UPV por su colaboración con el proyecto de innovación y mejora educativa PIME B-25.

\section{Referencias}

HEID, M.K., BLUME, G.W. (2008). “Technology and the development of algebraic understanding”. M.K. Heid, G.W. Blume (Eds.), Research on technology and the teaching and learning of mathematics: Research syntheses, vol. 1, Charlott, NC, p. 55-108.

STK. System Tool Kit. <http://www.agi.com/products/stk/> [Consulta: 15 de mayo de 2015]

TALL, D. (2000). “Cognitive development in advanced mathematics using technology”. Mathematics Education Research Journal, vol. 12, issue 3, p. 210-230.

TIANA, Y., LIU, H. YIN, J., LUO, M., WUA, G. (2015). "Evaluation of simulation-based training for aircraft carrier marshalling with learning cubic and Kirkpatrick's models". Chinese Journal of Aeronautics, vol. 28, issue 1, p. 152-163. < doi:10.1016/j.cja.2014.12.002>

TIWARI, S.R., NAFEES, L., KRISHNAN, O. (2014). "Simulation as a pedagogical tool: Measurement of impact on perceived effective learning”. The International Journal of Management Education, vol. 12, issue 3, p. 260-270. 